\title{
Produção de serapilheira em floresta Atlântica secundária numa paisagem fragmentada (Ibiúna, SP): importância da borda e tamanho dos fragmentos
}

\author{
MARIANA MORAIS VIDAL ${ }^{1}$, VÂNIA REGINA PIVELLO ${ }^{1,2}$, \\ SÉRGIO TADEU MEIRELLES ${ }^{1}$ e JEAN PAUL METZGER ${ }^{1}$
}

(recebido: 10 de novembro de 2006; aceito: 27 de outubro de 2007)

\begin{abstract}
Litter production in a fragmented Atlantic forest landscape (Ibiúna, SP): the importance of the edge and fragment size). Litter is an important compartment in tropical forests, responsible for most of nutrient cycling. Several environmental factors may affect litter production, and the ones mostly investigated are climate and vegetation structure. The influence of landscape structure on patterns of litter production is poorly studied. This study investigated edge and size

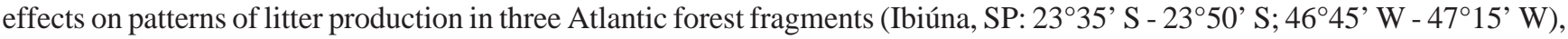
one large (ca. $175 \mathrm{ha}$ ) and two small (ca. $5 \mathrm{ha}$ ) with different degrees of isolation. The litter collected was fractioned following the predominant plant material, and grouped into two periods, of high and low production. We used analysis of variance and a posteriori tests to detect differences in litter production between edge and center of each fragment, and among the centers of the three fragments. Most biomass consisted of "leaves+flowers", followed by "twigs $\leq 0.5 \mathrm{~cm}$ " $(62.9 \%$ and $24.7 \%$ of the total), a common pattern in tropical forests. It was possible to perceive an edge effect related to litter production in the three fragments, especially in the large one, and higher litter production in their centers. The observed patterns showed to be strongly related to the vegetation structure, as the size of trees and canopy density. A higher production of "fruits+seeds" in the large fragment or in the small and connected one indicate a relationship between forest connectivity and the activity of disperser animals.
\end{abstract}

Key words - habitat fragmentation, landscape structure, litterfall, litter production, Plateau Atlantic forest

RESUMO - (Produção de serapilheira em floresta Atlântica secundária numa paisagem fragmentada (Ibiúna, SP): importância da borda e tamanho dos fragmentos). A serapilheira constitui um importante compartimento florestal, principal responsável pela ciclagem dos nutrientes. Diversos fatores podem afetar a produção de serapilheira, sendo os climáticos e a estrutura da vegetação os mais comumente investigados. Também a estrutura da paisagem pode influir nos padrões de produção da serapilheira, abordagem ainda muito pouco explorada. Este estudo objetivou avaliar efeitos de borda e de tamanho na produção de serapilheira em três fragmentos de Floresta Atlântica de Planalto (Ibiúna, SP: $23^{\circ} 35^{\prime} \mathrm{S}$ - 2350' S; $46^{\circ} 45^{\prime} \mathrm{W}-47^{\circ} 15^{\prime} \mathrm{W}$ ), um grande (aproximadamente $175 \mathrm{ha}$ ) e dois pequenos (aproximadamente $5 \mathrm{ha}$ ), com diferentes graus de isolamento. A serapilheira coletada foi separada em frações, conforme os órgãos vegetais predominantes, e em dois períodos, conforme maior ou menor produção. Foram utilizadas análises de variância e testes a posteriori para detectar diferenças na produção de serapilheira entre borda e interior de cada fragmento, e entre os interiores dos três fragmentos analisados. A maior biomassa foi de "folhas+flores", seguida de "caules $\leq 0,5 \mathrm{~cm}$ " (62,9\% e 24,7\% do total), padrão comum em florestas tropicais. Houve indicação de efeito de borda quanto à produção de serapilheira nos três fragmentos, especialmente no maior, com maior produção de material nos seus interiores. Os padrões verificados se relacionam fortemente a características da estrutura da floresta, como porte das árvores e desenvolvimento do dossel. A maior produção de "frutos+sementes" no fragmento grande e no pequeno conectado apontou para uma relação entre conectividade das manchas de mata e a visitação de animais dispersores.

Palavras chave - estrutura da paisagem, floresta Atlântica de planalto, fragmentação de habitats, serapilheira

\section{Introdução}

A serapilheira acumulada sobre o solo das florestas tem um papel importante na dinâmica desses ecossistemas. Nas florestas tropicais, em que predominam as cadeias tróficas de detritos, as transformações associadas à

\footnotetext{
1. Universidade de São Paulo, Instituto de Biociências, Departamento de Ecologia, Rua do Matão, Travessa 14, Cidade Universitária, 05508-900 São Paulo, SP, Brasil.

2._Autor para correspondência: vrpivel@usp.br
}

ciclagem dos nutrientes - que ocorrem no compartimento da serapilheira - concentram a maior parcela da energia que flui no sistema (Golley et al. 1978). A serapilheira também é um importante reservatório de nutrientes para as plantas, além de proteger o solo de forças erosivas, como chuvas (Ewell 1976, Moraes 2002). Padrões de produção e acúmulo da serapilheira introduzem também heterogeneidade temporal e espacial no ambiente, podendo afetar a estrutura e a dinâmica da comunidade de plantas, bem como diversos processos do ecossistema (Facelli \& Pickett 1991). Portanto, entender os padrões 
de produção da serapilheira é fundamental para a compreensão da dinâmica e do funcionamento dos ecossistemas, bem como para seu monitoramento.

Diversos são os fatores que podem influenciar a produção de serapilheira: clima, fertilidade do solo, composição de espécies na comunidade, estrutura da vegetação, estádio sucessional da floresta, perturbações antropogênicas na floresta e no entorno (Vitousek \& Sanford 1986, Songwe et al. 1988, Schlittler et al. 1993, Delitti 1995). A fragmentação dos habitats leva à criação de bordas abruptas, expostas a ambientes abertos, e pode modificar severamente as condições microclimáticas até centenas de metros para dentro da floresta, causando maior insolação, exposição ao vento e dessecamento (Lovejoy et al. 1984, Kapos 1989, Laurance 1991, Murcia 1995, Laurance et al. 1998, 2002). Essas condições, por sua vez, favorecem a mortalidade de árvores ou a queda de folhas e ramos, resultando num aumento temporário da produção de serapilheira (Lovejoy et al. 1984, Kapos 1989, Murcia 1995, Fox et al. 1997, Laurance et al. 1998, 2002, Fahrig 2003). Outra consequiência induzida pelas perturbações em bordas é o estabelecimento de espécies típicas dos estádios sucessionais iniciais, de crescimento rápido e vida relativamente curta, com madeira de baixa densidade (no caso de lenhosas), baixa resistência a predadores e a agentes infecciosos (Jordan 1985). Essas espécies produzem grande quantidade de fitomassa, e sua alta taxa de renovação acaba gerando grande massa de serapilheira nas bordas (Ewel 1976, Brown \& Lugo 1990, Oliveira-Filho et al. 1997, Gascon et al. 2000).

A fragmentação de habitats, além de criar bordas, leva à formação de manchas remanescentes de habitat - os fragmentos - com tamanhos variáveis e isoladas de outras manchas semelhantes, em maior ou menor grau. Tanto o tamanho do fragmento como seu grau de isolamento também podem afetar a produção de serapilheira. Vários autores (Lovejoy et al. 1984, Kapos 1989, Laurance et al. 1998, entre outros) encontraram, na Amazônia, uma intensificação dos efeitos de borda - notadamente a maior mortalidade de árvores - em fragmentos pequenos e isolados, quando comparados à floresta contínua. Isso ocorreu devido à maior quantidade relativa de bordas em fragmentos pequenos e, em relação ao isolamento, devido a mudanças locais no regime hídrico, causadas por alterações no uso das terras no entorno, e que repercutiram em todo o fragmento. $\mathrm{O}$ grau e a extensão desses efeitos foram proporcionais à distância a outras áreas florestadas (Kapos 1989).

Pelos fatos acima expostos, ao se comparar fragmentos florestais que se encontram na mesma situação climática e são provenientes do mesmo tipo vegetacional, espera-se que aqueles pequenos e isolados - portanto, com maior proporção de bordas e mais afetados pelo entorno - produzam maiores massas de serapilheira em relação aos maiores e com menor isolamento. Ainda, espera-se que as porções centrais dos fragmentos, por estarem mais protegidas dos efeitos de borda, tenham uma produção de serapilheira menor que as bordas, por possuírem menos espécies sucessionais iniciais (Lovejoy et al. 1984, Kapos 1989, Fox et al. 1997, Laurance et al. 2002).

Essas conseqüências da fragmentação - efeitos de borda, do tamanho e do isolamento do fragmento são tidas como dos mais importantes mecanismos causadores de mudanças em processos nos ecossistemas (Tabarelli et al. 1999), porém ainda são muito pouco explorados em Floresta Atlântica. Com a finalidade de avaliar efeitos de borda e de tamanho na produção de serapilheira em três fragmentos de Floresta Atlântica de Planalto, este estudo procurou averiguar as seguintes questões:

1) Há diferença na quantidade de serapilheira (total e de diferentes frações) produzida na borda e no interior de cada um dos três fragmentos florestais avaliados (efeito de borda)?

2) Há diferença na quantidade de serapilheira (total e de diferentes frações) produzida entre os interiores dos fragmentos de diferentes tamanhos, que são considerados suas porções mais preservadas e menos sujeitas aos efeitos de borda (efeito do tamanho)?

\section{Material e métodos}

Área de estudo - A área de estudo localiza-se no Município de Ibiúna (SP), sobre o Planalto Cristalino de Ibiúna (Serra de Paranapiacaba, Planalto Atlântico), em altitudes de 850 a 1.100

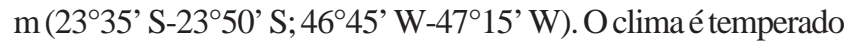
quente e úmido, $C f a$ (conforme Köppen 1948), com precipitação média anual em torno de $1.340 \mathrm{~mm}$, menor nos meses de abril a setembro, mas sem déficit hídrico (conforme dados de: Instituto Agronômico de Campinas- CIIAGRO s/data). Predominam na região os Argissolos Vermelho-Amarelos, Latossolos Vermelho-Amarelos e Cambissolos (Ross \& Moroz 1997), sobre os quais desenvolveu-se originalmente a floresta ombrófila densa montana (conforme Veloso et al. 1991). Grande parte da floresta original, porém, foi derrubada para o fornecimento de madeira e carvão e, posteriormente, para o estabelecimento de culturas agrícolas (Seabra 1971) e hoje, a vegetação natural remanescente é secundária, formada por fragmentos florestais com diferentes estádios de regeneração, entremeados a plantações de Eucalyptus spp., Pinus spp. e, principalmente, hortaliças e frutas. Isso resulta numa paisagem heterogênea, com grande variedade de fitofisionomias, que refletem a história de uso das terras. Apesar de alterada, a 
paisagem ainda mantém grande parte das espécies florestais (Pardini et al. 2005, Bernacci et al. 2006, Develey \& Metzger 2006), e está inserida na Reserva da Biosfera do Cinturão Verde da Cidade de São Paulo. Nessa paisagem, foram escolhidos os fragmentos florestais estudados.

Escolha dos fragmentos - Três fragmentos florestais foram selecionados, sendo dois pequenos (4,75 ha e 5,48 ha) e um grande $(175,10$ ha). Procurou-se fragmentos com estrutura florestal (dada pela estratificação, porte das árvores e formas de vida presentes) visualmente semelhante e com estádios sucessionais e históricos de perturbação também similares. Desta forma, foram desconsiderados fragmentos que apresentavam perturbações humanas recentes mais intensas, tais como corte ou uso do sub-bosque, entrada de gado, indícios de fogo ou trilhas em grande número ou de grande porte.

Os fragmentos selecionados também possuíam a mesma situação de entorno, que era predominantemente agrícola. A partir de fotografias aéreas de 1962, 1981 e 2000, foi ainda possível determinar a idade das matas com uma precisão de cerca de 5 anos (A.M.G. Teixeira e J.P. Metzger, dados nãopublicados). Assim, os três fragmentos selecionados eram predominantemente formados por matas com pelo menos 60 anos de regeneração. Entretanto, propositalmente, os fragmentos escolhidos apresentavam características diferentes no contexto da paisagem: um dos fragmentos pequenos (fragmento 1) era totalmente isolado de outras manchas florestais por áreas agrícolas, enquanto o outro fragmento pequeno (fragmento 2) era conectado por meio de um corredor florestal ao fragmento grande (fragmento 3) (figura 1).

Coleta da serapilheira - Foram instalados 54 coletores circulares (área interna $=0,5 \mathrm{~m}^{2}$, profundidade do bojo $\approx 15$ $\mathrm{cm}$ ), confeccionados com tela de nylon (malha $=1 \mathrm{~mm})$ e suspensos a 80-90 $\mathrm{cm}$ do solo por meio de tubos de PVC. Cada fragmento recebeu 18 coletores, tendo sido nove

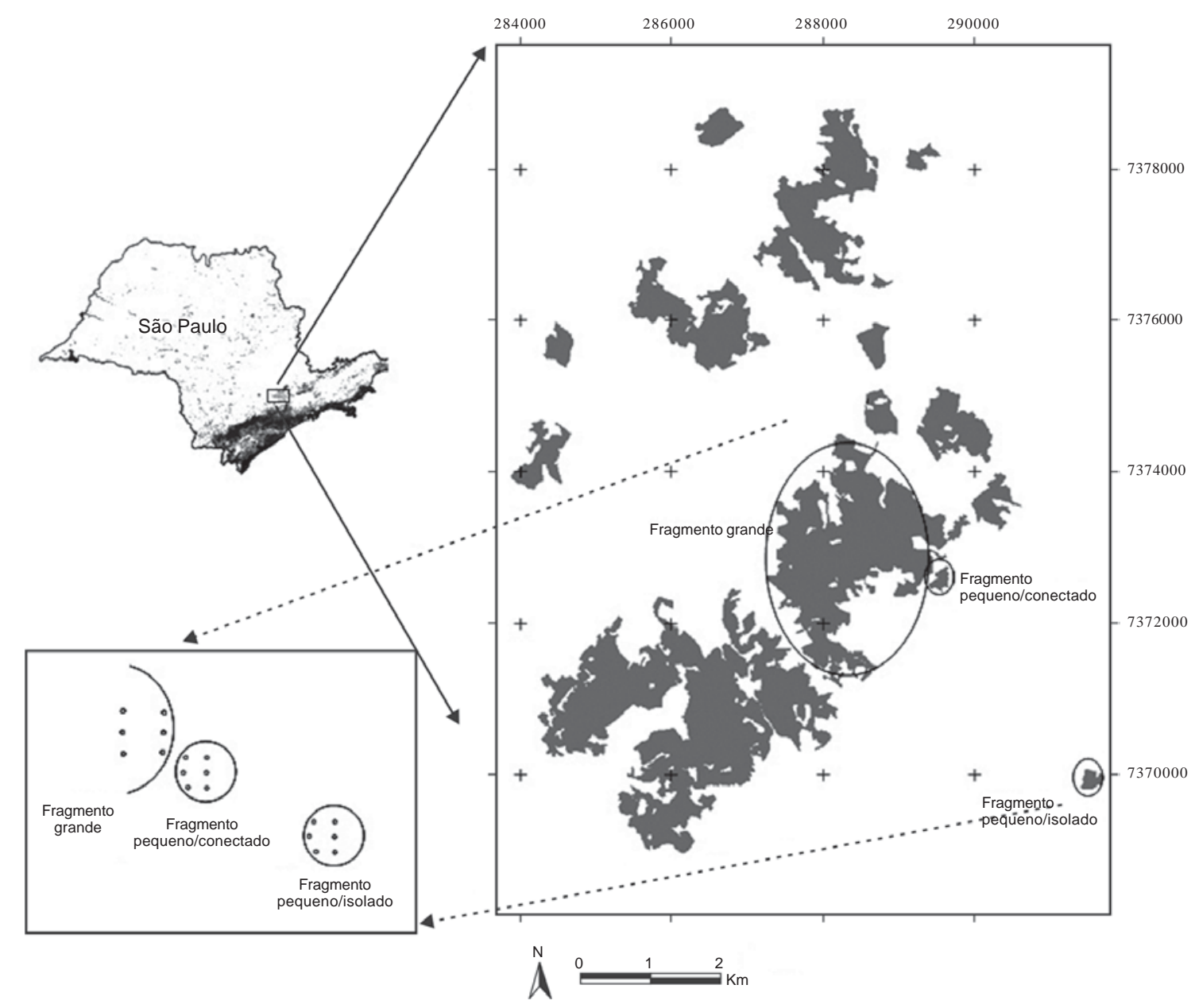

Figura 1. Área de estudo (Planalto de Ibiúna, SP), com a localização dos fragmentos florestais estudados (círculos) e indicação aproximada dos pontos de coleta, representados por pequenos círculos. Cada pequeno círculo representa um grupo de três coletores. A escala e o formato dos trios de coletores e dos fragmentos florestais são de caráter meramente ilustrativo. (Modificado de Silva et al. 2007.)

Figure 1. The study area, at Ibiúna Plateau, SP: schematic location of the studied fragments (circles) and of the sampling points (represented by small circles). Each small circle represents a group of three litter traps. Fragment and litter trap form and scale are merely illustrative. (Modified from Silva et al. 2007.) 
dispostos a $10 \mathrm{~m}$ da borda do fragmento e nove no centro, arranjados em trios, que passaram a ser as unidades amostrais (figura 1). A borda do fragmento era determinada por estradas ou por mudanças abruptas no uso das terras. No caso dos fragmentos pequenos, os coletores instalados no centro distavam cerca de 50-60 m da borda e, no caso do fragmento grande, cerca de $400 \mathrm{~m}$ da borda.

Os dados foram obtidos em 10 períodos de coleta, em intervalos de $35 \pm 8$ dias, entre março de 2001 e janeiro de 2002 (06/mar/2001-06/abr/2001; 06/abr/2001-03/mai/2001; 03/ mai/2001-31/mai/2001;31/mai/2001-13/jul/2001; 13/jul/2001-10/ ago/2001; 10/ago/2001-12/set/2001; 12/set/2001-11/out/2001; 11/out/2001-13/nov/2001; 13/nov/2001-11/dez/2001; 11/dez/ 2001-09/jan/2002). O material depositado nos coletores foi seco a $60{ }^{\circ} \mathrm{C}$, até peso constante, e separado nas frações: "caules $\leq 0,5 \mathrm{~cm}$ " (caules com diâmetro igual ou inferior a $0,5 \mathrm{~cm}$ ), "caules > 0,5cm" (caules com diâmetro entre $0,6 \mathrm{~cm}$ e $1,5 \mathrm{~cm}$ ), "folhas+flores", "frutos+sementes" e "detritos", tendo sido as partes animais incluídas nesta última fração. Cada fração foi pesada separadamente.

Análise dos dados - O conjunto das coletas foi arbitrariamente separado em duas classes, que representaram aproximadamente períodos de maior e menor produção de serapilheira, respectivamente de agosto/2001 a janeiro/2002 e de março/2001 a julho/2001. Esse procedimento teve o objetivo de simplificar as análises, uma vez que não era intenção avaliar a variação sazonal de produção da serapilheira. As análises foram feitas com base nesses dois períodos e também considerando os valores totais (soma dos dois períodos).

A fim de comparar a produção de serapilheira na borda e no interior de cada um dos três fragmentos individualmente (ou seja, verificar efeito de borda em cada fragmento), bem como comparar a produção de serapilheira entre os interiores dos três fragmentos (ou seja, comparar os fragmentos por meio de suas partes menos perturbadas), os valores das frações "caules $\leq 0,5 \mathrm{~cm}$ " e "folhas+flores" foram comparados, após transformações logarítmicas, por meio de análise de variância unidirecional (one-way ANOVA) e teste Dunnett C (Zar 1999). No caso da fração "frutos+sementes", cujos valores não apresentaram distribuição normal, as mesmas comparações foram feitas pelo teste de Kruskal-Wallis (Zar 1999). Os detritos foram excluídos das análises por fornecerem pouca informação biológica, já que tratam de uma fração composta por material impossibilitado de ser identificado e de diferentes origens. Os caules com diâmetro $>0,5 \mathrm{~cm}$ foram relativamente raros e por isso também não foram estatisticamente analisados.

Caracterização dos fragmentos - A fim de se obter subsídios para a investigação das causas provavelmente envolvidas nos padrões de produção de serapilheira, foram quantificados alguns atributos dos fragmentos, bem como da estrutura de suas matas.

Para caracterizar a situação dos fragmentos na paisagem, utilizou-se um mapa de uso e cobertura das terras, produzido por interpretação de fotografias aéreas (abril/2000), na escala 1:10.000 (Silva et al. 2007), a partir do qual foram calculadas: a área total de cada fragmento (ha); a área nuclear (ha) - que é a área do fragmento excluindo-se bordas de $50 \mathrm{~m}$ (segundo Murcia 1995); a proximidade de fragmentos florestais (PROX) - resultante da somatória da razão da área de fragmentos vizinhos $\left(\mathrm{m}^{2}\right)$ pelo quadrado da distância borda-a-borda entre o fragmento focal e o fragmento vizinho, num raio de $800 \mathrm{~m}$ a partir da borda do fragmento (segundo McGarigal \& Marks 1995); a distância de cada fragmento a outros fragmentos florestais maiores que 50 ha (ISO). A vizinhança de $800 \mathrm{~m}$ foi escolhida, a fim de incluir os principais remanescentes do entorno imediato dos fragmentos focais e que teriam mais chance de contribuir para a chuva de sementes.

Para caracterizar a estrutura da vegetação no interior dos fragmentos, foram utilizados alguns parâmetros, medidos por L. Alves e J. P. Metzger (dados não publicados): densidade, dada pelo número médio de indivíduos arbóreos com diâmetro à altura do peito $(\mathrm{DAP})>5 \mathrm{~cm}$ em 13 parcelas de 5 por $5 \mathrm{~m}$; altura média (ALT) de árvores com DAP > 5 cm (100 árvores amostradas para os fragmentos pequenos e 400 para o fragmento grande); abertura média do dossel, resultante de 52 medidas obtidas com esfero-densiômetro, em pontos aleatórios no interior de cada fragmento. Dados florísticos (não publicados) obtidos por L.C. Bernacci, G. Durigan, G.A.D.C. Franco, G.F Arbocz e E.L.M Catharino também foram utilizados para caracterizar os grupos funcionais das espécies presentes no interior dos fragmentos, com base no nicho de regeneração característico da espécie (100 árvores com DAP $>5 \mathrm{~cm}$ amostradas para os fragmentos pequenos e 400 para o fragmento grande).

\section{Resultados}

Produção de serapilheira nos fragmentos ao longo do período - A produção total de serapilheira em cada fragmento, nos dez meses de coleta (entre março/2001 e janeiro/2002), foi de 75,17 $\pm 41,20 \mathrm{~g} \mathrm{~m}^{-2}$ e 88,06 \pm $53,93 \mathrm{~g} \mathrm{~m}^{-2}$, respectivamente na borda e no interior do fragmento 1; de $68,73 \pm 33,25 \mathrm{~g} \mathrm{~m}^{-2} \mathrm{e} 72,20 \pm 27,99 \mathrm{~g} \mathrm{~m}^{-2}$, respectivamente na borda e no interior do fragmento 2 , e de 49,02 $\pm 21,83 \mathrm{~g} \mathrm{~m}^{-2} \mathrm{e} 68,11 \pm 41,18 \mathrm{~g} \mathrm{~m}^{-2}$, respectivamente na borda e no interior do fragmento 3 . A maior produção ocorreu no fragmento 1 , seguida do fragmento $2 \mathrm{e}$, por fim, do fragmento 3, em ambos os períodos analisados, e sempre maior nos interiores dos três fragmentos.

Entre agosto/2001 e janeiro/2002, a produção de serapilheira foi maior, e menor entre março/2001 e julho/ 2001 (figura 2), considerando-se tanto o total como as frações foliares e caulinares. A produção de "frutos+sementes" apresentou certa constância ao longo do período.

A fração "folhas+flores", composta majoritariamente por folhas, foi a que apresentou maior massa dentre todas as frações, participando em $62,9 \%$ da serapilheira total; a fração "caules $\leq 0,5 \mathrm{~cm}$ " foi a segunda a mais contribuir para a serapilheira, correspondendo a $24,7 \%$ 

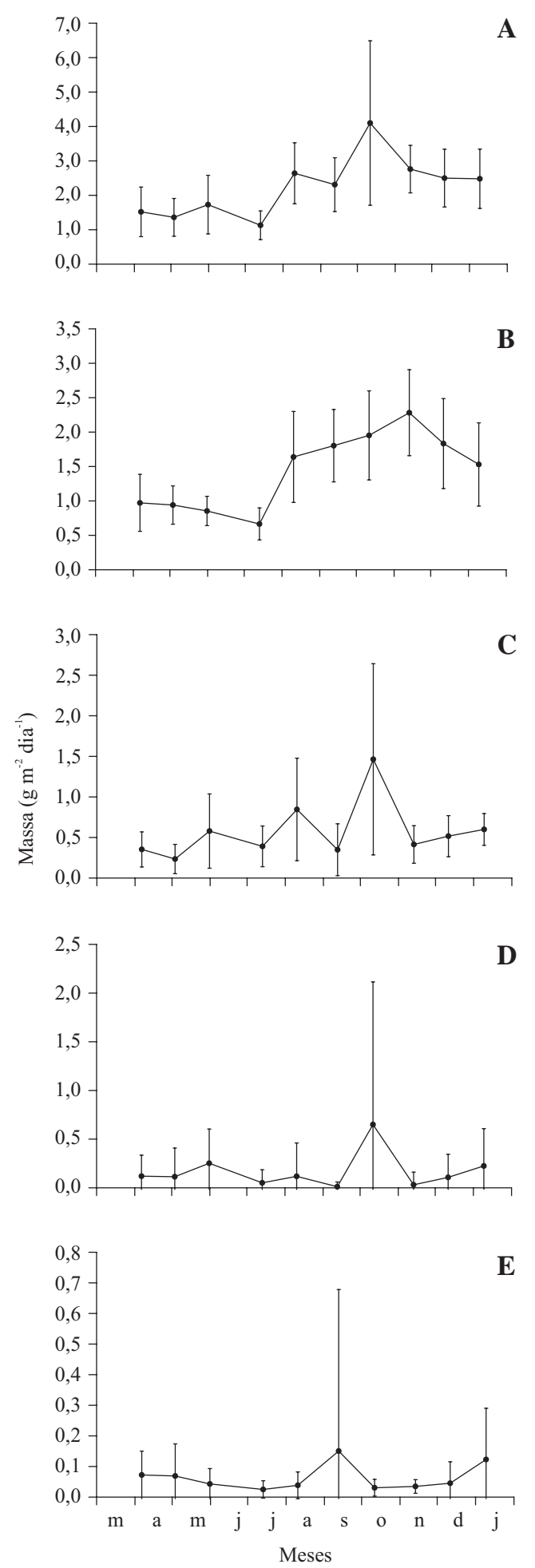

Figura 2. Valores médios diários ( $\mathrm{g} \mathrm{m}^{-2} \mathrm{dia}^{-1} \pm$ desvio padrão) de diferentes frações da serapilheira produzida entre março de 2001 e janeiro de 2002, em Ibiúna, SP. A. Total. B. Folhas e flores. C. Caules $\leq 0,5 \mathrm{~cm}$. D. Caules $>0,5 \mathrm{~cm}$. E. Frutos e sementes.

Figure 2. Mean daily values $\left(\mathrm{g} \mathrm{m}^{-2} \mathrm{day}^{-1} \pm\right.$ standard deviation) of litter fractions produced from March/2001 to January/2002, in Ibiúna, SP. A. Total. B. Leaves and flowers. C. Stems $\leq 0,5 \mathrm{~cm}$. D. Stems $>0,5 \mathrm{~cm}$. E. Fruits and seeds. do total; os caules maiores (diâmetros entre 0,6 e 1,5 $\mathrm{cm}$ ) participaram com 7,0\% da massa total; a categoria "frutos+sementes" contribuiu com $2,8 \%$ e, por fim, os "detritos", com 2,6\% do total.

O padrão geral de maior contribuição da fração "folhas+flores" na serapilheira, seguida das frações caulinares, se repetiu em cada fragmento (figura 3). No período correspondente à maior produção, a fração "folhas+flores" foi proporcionalmente maior do que no período de menor produção, nos fragmentos pequenos (1 e 2). Já a maior variação nas frações caulinares não foi entre fragmentos diferentes, mas entre os períodos de maior e menor produção de folhedo, no fragmento 2 (figura 3).
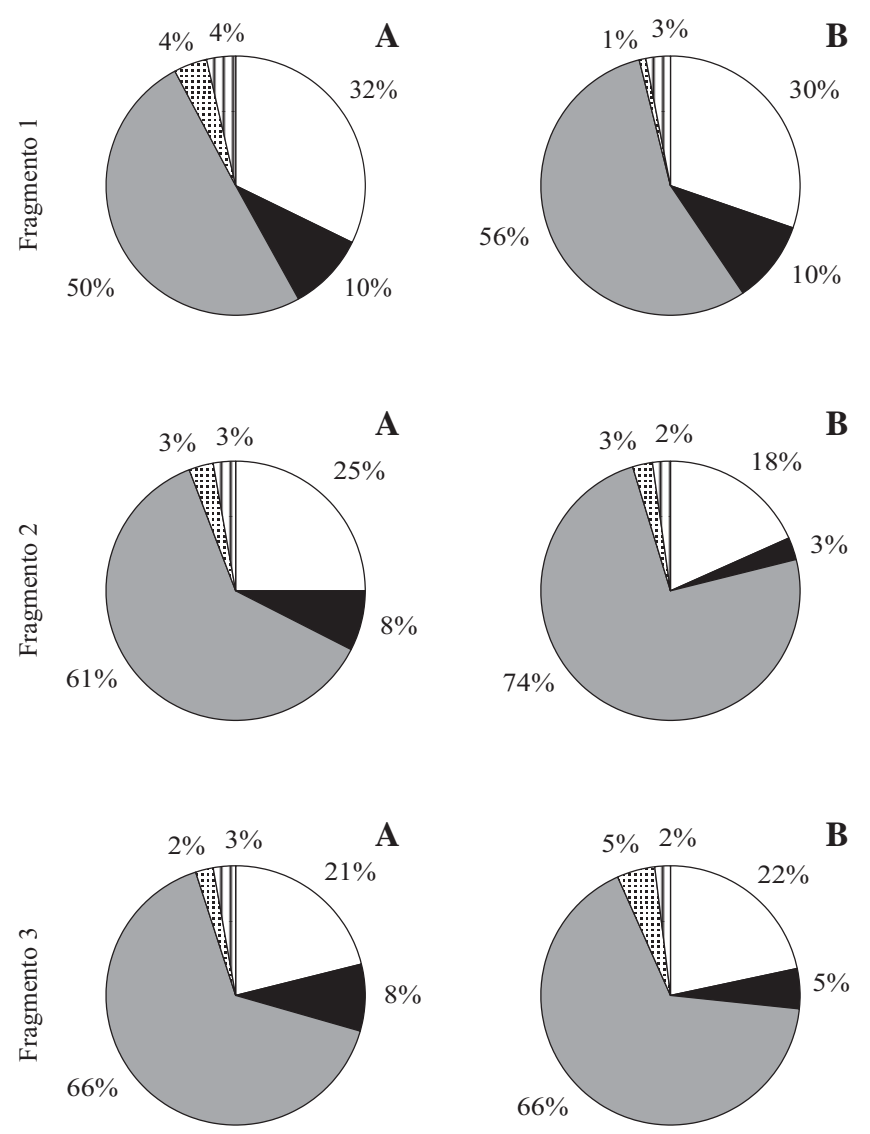

Figura 3. Porcentagens das diferentes frações da serapilheira coletadas em cada fragmento analisado, nos períodos de menor (A) e maior (B) produção de serapilheira (Ibiúna, SP). $($ Branco $=$ "caules $\leq 0,5 \mathrm{~cm}$ "; preto $=$ "caules $>0,5 \mathrm{~cm}$ "; cinza $=$ "folhas+flores"; pontilhado = "frutos+sementes"; hachurado = "detritos").

Figure 3. Percentages of litter fractions collected in the studied fragments, in the periods of low (A) and high (B) production (Ibiúna, SP) (White = "twigs $\leq 0,5 \mathrm{~cm}$ "; black = "branches > $0,5 \mathrm{~cm}$ "; grey = "leaves+flowers"; spotted = "fruits+seeds"; hatched = "debris"). 
Produção de serapilheira no interior e na borda de cada fragmento - Comparando-se a produção de serapilheira (menos detritos) entre a borda e o interior de cada um dos três fragmentos (figura 4A), tem-se significativamente maior produção no interior do fragmento 2 (Dunnett, $P$ $<0,05)$ para o período de menor produção de material $\left(F_{g l=23}=6,578 ; n=6 ; P=0,018\right)$, bem como significativamente maior produção de serapilheira no interior do fragmento 3 , para ambos os períodos considerados $\left(F_{g l=23}=5,136 ; P=0,034 ; F_{g l=35}=4,222\right.$; $P=0,048$, respectivamente para os períodos de menor e maior produção; $\mathrm{n}=6$ ) (Dunnett, $P<0,05$ ).

Considerando-se a fração "folhas+flores", foi verificada uma produção significativamente maior no interior do fragmento 1 , no período de maior produção de serapilheira $\left(F_{g l 35}=5,863 ; n=6 ; P=0,021\right)$ (Dunnett, $P<0,05)$. Já o fragmento 2 apresentou maior quantidade de caules finos em seu interior do que na borda, no período de menor produção $\left(F_{g l=23}=4,802 ; n=6 ; P=0,039\right)$ (Dunnett, $P<0,05$ ). O fragmento 3, por sua vez, apresentou diferenças significativas entre borda e interior para a fração de caules finos, em ambos os períodos de produção de serapilheira (Dunnett, $P<0,05$ ), com maior produção dessa fração no seu interior $\left(F_{g l=23}=7,850\right.$; $P=0,010 ; F_{g l=35}=12,029 ; P=0,001 ;$ respectivamente para os períodos de menor e maior deposição de material; $n=6$ ) (Dunnett, $P<0,05$ ). O fragmento 3 também produziu quantidade significativamente maior de frutos e sementes em seu interior, no período de maior produção de material $\left(H_{g l=1}=4,230 ; P=0,040\right)$ (Dunnett, $\left.P<0,05\right)$ (figuras 4B, 4C, 4D).

Produção de serapilheira nos interiores dos fragmentos - O interior do fragmento 3 diferiu do fragmento 1 (pequeno) quanto à produção total de serapilheira, com
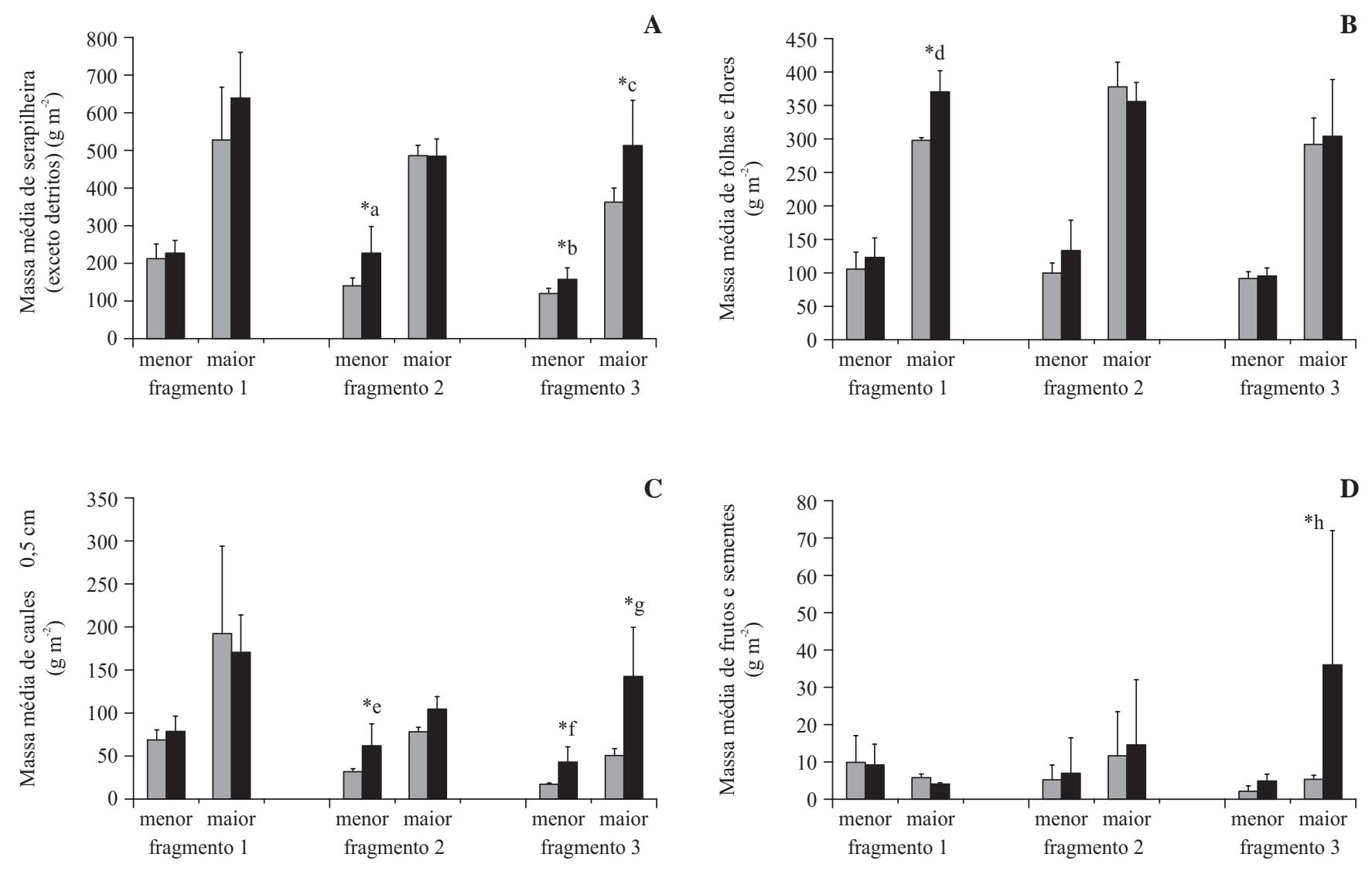

Figura 4. Produção de serapilheira entre mar/2001 e jan/2002 na borda e no interior de cada fragmento analisado (g m²; média \pm desvio padrão), nos períodos de maior e menor produção de material. A. Serapilheira total menos detritos. B. Fração "folhas+flores". C. Fração "caules $\leq 0,5$ cm". D. Fração "frutos+sementes". (* = diferenças significativas; cinza = borda; preto = interior).

Figure 4. Production of litter from March/2001 to January/2002 in the edge and center of each studied fragment $\left(\mathrm{g} \mathrm{m}^{-2} ; \mathrm{mean}^{ \pm}\right.$ standard deviation), in the periods of low and high production. A. Total litter excluding debris. B. "Leaves+flowers". C. "Twigs $\leq 0,5 \mathrm{~cm}$ ". D. "Fruits+seeds". (* = significant differences; grey = edge; black = center). 
valor significativamente menor (Dunnett, $P<0,05$ ) no período de menor produção de material $\left(F_{g l=35}=3,324\right.$; $n=9 ; P=0,048)$. As frações foliares e caulinares não diferiram significativamente entre os interiores dos três fragmentos, mas verifica-se que o fragmento 1 produziu menos "frutos+sementes" no período de maior produção de serapilheira $\left(H_{g l=2}=6,612 ; n=9 ; P=0,037\right)$ (figura 5), distinguindo-se dos demais (Dunnett, $P<0,05$ ). Caracterização dos fragmentos - $\mathrm{O}$ fragmento 3 , com área total de 175,10 ha, apresentou cerca de 118 ha de área nuclear (tabela 1). Os fragmentos 1 e 2, devido à sua pequena área total (cerca de $5 \mathrm{ha}$ ), apresentaram pouca área nuclear ( $\leq 1$ ha). Os índices PROX e ISO confirmaram a baixa proximidade do fragmento 1 a outros remanescentes florestais de maior porte (> 50 ha), contrariamente ao fragmento 2 (tabela 1 ).

A altura média das árvores variou entre 10,0 e 11,4 $\mathrm{m}$ entre os fragmentos, e a abertura média do dossel,

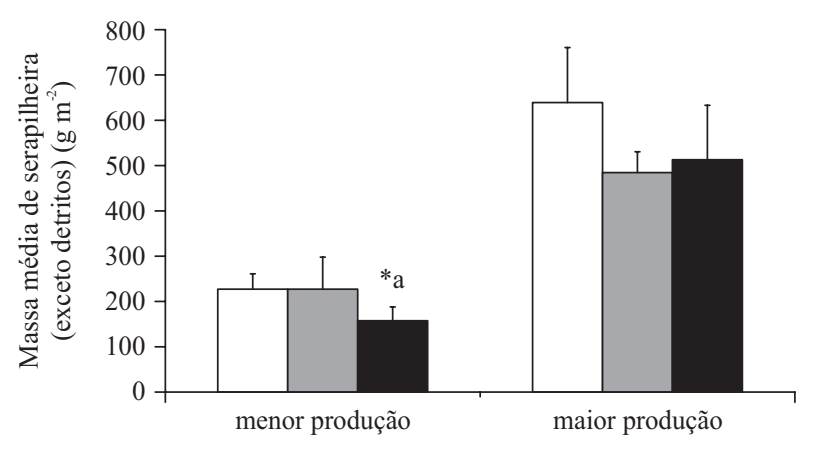

A
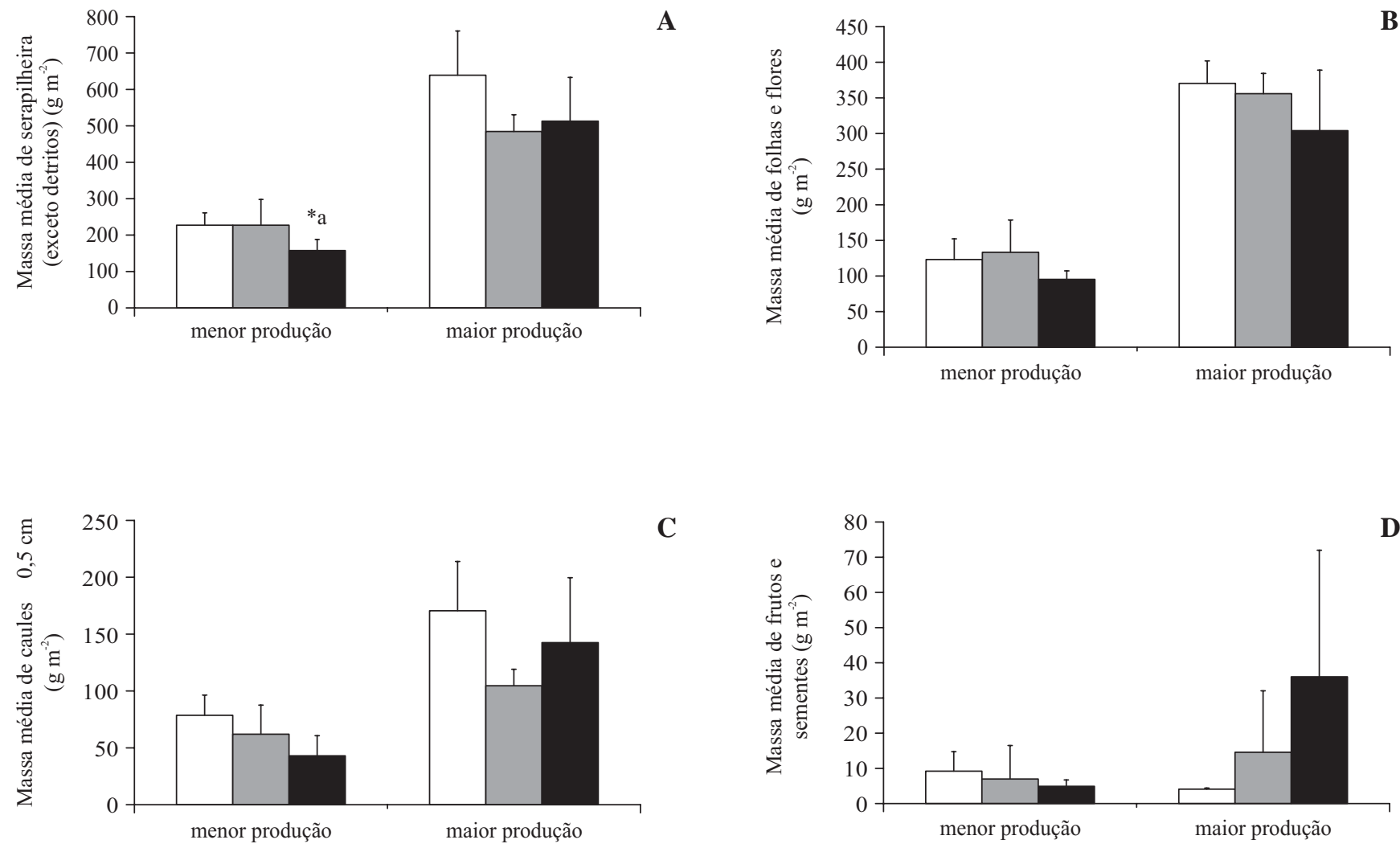

C

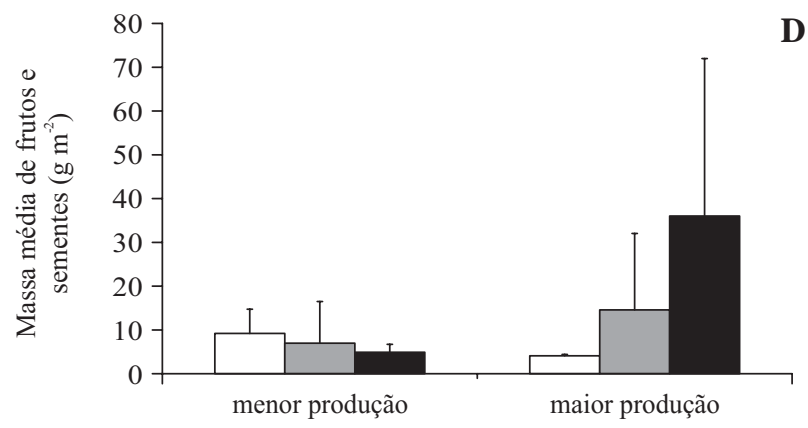

Figura 5. Serapilheira produzida entre mar/2001 e jan/2002 no interior dos fragmentos analisados (média \pm desvio padrão), nos períodos de maior e menor produção de material. A. Serapilheira total menos detritos. B. Fração "folhas+flores". C. Fração "caules $\leq 0,5 \mathrm{~cm}$ ". D. Fração "frutos+sementes". (* = diferenças significativas; branco = fragmento 1; cinza = fragmento 2; preto $=$ fragmento 3 ).

Figure 5. Litter produced from March/2001 to January/2002 in the center of each studied fragment (mean \pm standard deviation), in the periods of low and high production. A. = Total litter excluding debris. B. "Leaves+flowers". C. "Twigs $\leq 0,5 \mathrm{~cm}$ ". D. "Fruits+seeds". $(*=$ significant differences; white $=$ fragment 1 ; grey $=$ fragment 2 ; black $=$ fragment 3 ). 
Tabela 1. Parâmetros referentes à configuração espacial e à estrutura da vegetação dos fragmentos estudados (Ibiúna, SP). PROX = índice de proximidade; $\mathrm{ISO}=$ índice de isolamento; $\mathrm{ALT}=$ altura de árvores com DAP $>5 \mathrm{~cm}$ (média \pm desvio padrão); Arv $>5 \mathrm{~cm} \mathrm{ha}^{-1}=$ número de árvores com DAP $>5 \mathrm{~cm}$, por hectare (média \pm desvio padrão); Abertura $=$ porcentagem de abertura do dossel (média \pm desvio padrão).

Table 1. Some parameters related to spatial configuration and vegetation structure of the analyzed fragments (Ibiúna, SP). $\mathrm{PROX}=$ proximity index; ISO $=$ isolation index; ALT $=$ height of trees with diameter at breast height $(\mathrm{DBH})>5 \mathrm{~cm}(\mathrm{mean} \pm$ standard deviation); Arv $>5 \mathrm{~cm} \mathrm{ha}^{-1}=$ number of trees with $\mathrm{DBH}>5 \mathrm{~cm}$, per hectare (mean \pm standard deviation); Abertura $=$ percentage of canopy openness (mean \pm standard deviation).

\begin{tabular}{clccccccc}
\hline Fragmento & $\begin{array}{l}\text { Tamanho/ } \\
\text { conectividade }\end{array}$ & $\begin{array}{c}\text { Área total } \\
\text { (ha) }\end{array}$ & $\begin{array}{c}\text { Área nuclear } \\
\text { (ha) }\end{array}$ & PROX & $\begin{array}{c}\text { ISO } \\
(\mathrm{m})\end{array}$ & $\begin{array}{c}\text { ALT } \\
(\mathrm{m})\end{array}$ & $\begin{array}{c}\text { Arv }>5 \mathrm{~cm} \mathrm{ha}^{-1} \\
\left.\text { (indivíduos ha }^{-1}\right)\end{array}$ & $\begin{array}{c}\text { Abertura } \\
(\%)\end{array}$ \\
\hline 1 & $\begin{array}{l}\text { pequeno/ } \\
\text { isolado } \\
\text { pequeno/ }\end{array}$ & 5,48 & 1,08 & 51,9 & 1210 & $11,36 \pm 4,07$ & $1046,1 \pm 504,3$ & $0,88 \pm 0,50$ \\
2 & 4,75 & 0,79 & 850,7 & 45 & $11,01 \pm 3,1$ & $1200,0 \pm 632,5$ & $1,37 \pm 0,86$ \\
3 & $\begin{array}{l}\text { conectado } \\
\text { grande }\end{array}$ & 175,10 & 117,91 & 1721,1 & 0 & $10,03 \pm 3,9$ & $1830,8 \pm 839,6$ & $1,63 \pm 2,35$ \\
\hline
\end{tabular}

Tabela 2. Proporção das espécies e de indivíduos pertencentes a diferentes grupos funcionais conforme a guilda de regeneração típica da espécie, nos três fragmentos estudados. Maiores detalhes no texto.

Table 2. Proportion of species and individuals of different functional groups according to the typical regeneration guild, in the analyzed fragments. More details in the text.

\begin{tabular}{|c|c|c|c|c|c|}
\hline \multirow[t]{2}{*}{ Fragmento } & pioneira & $\begin{array}{l}\text { secundária } \\
\text { inicial }\end{array}$ & $\begin{array}{c}\text { secundária } \\
\text { tardia }\end{array}$ & umbrófila & $\begin{array}{c}\text { sem } \\
\text { classificação }\end{array}$ \\
\hline & \multicolumn{5}{|c|}{ Grupo funcional (\% de espécies) } \\
\hline 1 & 2,1 & 27,1 & 45,8 & 20,8 & 4,2 \\
\hline 2 & 14,3 & 50,0 & 23,8 & 11,9 & 0,0 \\
\hline \multirow[t]{2}{*}{3} & 7,9 & 51,1 & 27,3 & 12,5 & 1,1 \\
\hline & \multicolumn{5}{|c|}{ Grupo funcional (\% de indivíduos) } \\
\hline 1 & 1,0 & 28,0 & 45,0 & 19,0 & 7,0 \\
\hline 2 & 12,0 & 54,0 & 15,0 & 19,0 & 0,0 \\
\hline 3 & 4,2 & 57,7 & 16,0 & 21,2 & 0,7 \\
\hline
\end{tabular}

\section{Discussão}

Assim como neste estudo, também em outras florestas tropicais foi observada uma grande contribuição da fração foliar na serapilheira, em comparação com as demais frações (Golley et al. 1978, Veneklaas 1991). No domínio Atlântico Moraes (1993) e Moraes et al. (1999) verificaram na Ilha do Cardoso, Cananéia (floresta pluvial, SP), uma contribuição de $70 \%$ de folhas de serapilheira total, $15 \%$ de ramos, $13 \%$ de órgãos de reprodução e $2 \%$ de detritos. No Parque Estadual das Fontes do Ipiranga (floresta semidecídua, SP) e em Paranapiacaba (floresta pluvial, SP), respectivamente
Moraes (2002) e Domingos et al. (1997) encontraram contribuição da fração foliar por volta de $70 \%$, mas uma maior contribuição de galhos finos, em torno de $20 \%$. Essas variações nas proporções das frações da serapilheira podem estar ligadas a condições de pluviosidade e umidade do ar, à estrutura da floresta ou a situações de alteração ou degradação local, que acabam se refletindo na deciduidade da vegetação e nas taxas de ciclagem do material (Schlittler et al. 1993, Delitti 1995).

Neste estudo, houve maior produção da fração foliar durante o período úmido. Entretanto, o pico de produção de elementos caulinares coincidiu com o fim da estiagem e as fortes chuvas que marcam o início da estação úmida 
(setembro/outubro). As primeiras chuvas fortes após a estação seca promovem a queda de muitos ramos secos, que ainda permaneciam unidos à planta. Esse pico de produção de serapilheira, especialmente de ramos, após tempestades acompanhadas de ventos fortes também foi reportado por outros autores (Green 1998, Moraes 2002). Esse efeito foi mais pronunciado no fragmento 1 , talvez por sua situação de maior exposição aos fatores externos, devido à condição de maior isolamento na paisagem.

Outros autores também identificaram dois períodos distintos de produção de serapilheira. Moraes (2002) obteve um padrão muito semelhante ao aqui encontrado: maior produção entre setembro e janeiro, e menor entre março e julho. Em florestas tropicais, existe uma tendência de maior produção de serapilheira na época de maior restrição hídrica às plantas, para as florestas mais secas (florestas estacionais), enquanto que, à medida que o ambiente tem menos restrição hídrica, o período de maior produção de serapilheira vai se deslocando para a estação chuvosa (Cesar 1993, Green 1998, Moraes et al. 1999).

A produção geralmente menor de serapilheira nas bordas dos fragmentos, ora relacionada a um tipo de fração, ora a outro, evidenciou a existência de um efeito de borda referente à massa de serapilheira produzida. A acentuação desse efeito no fragmento 3 provavelmente se relaciona ao seu grande tamanho e à existência de uma área nuclear efetiva, enquanto que os fragmentos pequenos praticamente nem chegam a ter área nuclear, como também observado por outros autores, em fragmentos menores que 10 ha, por meio de outros parâmetros (Benitez-Malvido 1998, Tabarelli et al. 1999).

Entretanto, a tendência de menor produção de serapilheira nas bordas dos fragmentos revelou um padrão contrário ao esperado, levando-se em conta a situação, geralmente reportada para as bordas, de maior perturbação e maior dessecamento, resultando em menor taxa de decomposição e maior proporção de espécies dos estádios sucessionais iniciais (Lovejoy et al. 1984, Kapos 1989, Murcia 1995, Fox et al. 1997, OliveiraFilho et al. 1997, Sizer et al. 2000, Laurance et al. 2002). Assim como neste estudo, Didham \& Lawton (1999) também encontraram menor produção de serapilheira nas bordas de fragmentos da floresta Amazônica e afirmaram que os padrões referentes à produção e deposição de serapilheira são complexos e podem ser, em certos casos, mais relacionados a processos biológicos e à estrutura da floresta do que aos efeitos de borda derivados de alterações microclimáticas. Sizer et al.
(2000) verificaram estabilização na produção de serapilheira em bordas de floresta Amazônica poucos anos após a criação dessas bordas. Ewell (1976) afirma que, em florestas jovens e em áreas recém-perturbadas, a proporção de espécies de fases sucessionais iniciais aumenta a produção de serapilheira, mas esse efeito deixa de ser importante após 2 ou 3 décadas.

Os fragmentos aqui analisados são provenientes de processos de regeneração iniciados há mais de 60 anos, diferentemente da maioria dos fragmentos florestais estudados na Amazônia (Lovejoy et al. 1984, Kapos 1989, por exemplo), criados muito mais recentemente pela fragmentação da floresta primária. Esse histórico é percebido ainda hoje pela alta porcentagem de espécies secundárias iniciais e tardias no interior dos três fragmentos estudados (Bernacci et al. 2006). Apesar das conseqüências dos efeitos microclimáticos de borda poderem perdurar por algumas décadas (Oliveira-Filho et al. 1997, Laurance et al. 2002, Hill \& Curran 2003), a origem dos fragmentos aqui estudados, sua idade e sua composição predominante por espécies secundárias são prováveis fatores que fazem com que a estrutura da floresta passe a ter um papel mais importante na determinação de padrões na serapilheira do que o contraste microclimático entre borda e interior, assim como encontraram Didham \& Lawton (1999).

A estrutura da floresta guarda forte relação com a produção de serapilheira. Songwe et al. (1988) e Schlittler et al. (1993) verificaram uma relação direta entre a produção de serapilheira e o desenvolvimento do dossel. O porte grande das árvores, refletido num dossel bem desenvolvido, pode ser mais importante na produção de serapilheira do que a abundância de espécies pioneiras, como verificado por Werneck et al. (2001). Neste estudo, o fragmento 1 foi o que produziu maior massa total de serapilheira no período analisado e também produziu significativamente mais folhas e flores em seu interior, no período de maior produção de material. Isso pode ser explicado por sua estrutura de mata mais desenvolvida, com árvores de grande porte, dossel mais fechado, maior quantidade de epífitas e mais espécies tardias, geralmente de maior porte. Por outro lado, os interiores dos fragmentos $2 \mathrm{e} 3$ produziram significativamente mais caules finos do que as bordas. São fragmentos com maior proporção de espécies de estádios sucessionais iniciais, maior densidade de árvores finas e com dossel mais aberto, podendo estar sujeitos à maior penetração de vento e menor umidade interior (Kapos 1989, Ashton 1992), o que poderia resultar em maior produção e queda de ramos. 
No interior do fragmento 3, foi também verificada uma produção muito superior de frutos e sementes em relação à borda, na classe correspondente à maior deposição de material. Uma grande massa de frutos e sementes reflete mais a produção das espécies zoocóricas, as quais produzem frutos maiores e mais pesados. De fato, Pivello et al. (2006), analisando a chuva de sementes nos mesmos locais deste estudo, verificaram uma predominância de espécies zoocóricas no interior desse fragmento grande. Tabarelli et al. (1999) verificaram, em fragmentos de floresta Atlântica próximos aos deste estudo, uma relação direta entre o tamanho do fragmento e a importância de famílias de árvores produtoras de frutos carnosos, dispersos por vertebrados. Importantes dispersores, como primatas, pequenos marsupiais e aves de sub-bosque, são sensíveis à fragmentação, desaparecendo de fragmentos menores que 100 ha (Laurance 1991, Laurance et al. 2002). Ao contrário, em fragmentos com efetiva área interior, esses animais promovem o fluxo de diásporos zoocóricos, colaborando na manutenção dessa sinúsia.

Comparando-se a produção de serapilheira nos interiores dos três fragmentos, verificou-se que, no período de maior produção de serapilheira, foi significativamente mais baixa a quantidade da fração "frutos+sementes" no interior do fragmento $1 \mathrm{em}$ relação aos outros, havendo também uma tendência a um gradiente no aumento na deposição de frutos e sementes do fragmento 1 para o 2 e, por fim, para o 3 . Esse gradiente aponta para uma possível relação com o grau de conectividade dos fragmentos, sendo que, naqueles com maior conectividade, a visitação de animais dispersores é facilitada, o que promove a permanência de espécies zoocóricas nesses locais. A menor produção total de serapilheira no interior do fragmento grande, comparado aos pequenos, também pode estar relacionada com sua estrutura de mata mais aberta e mais baixa.

Concluindo, os dados obtidos neste estudo apontam para uma forte contribuição da estrutura da vegetação na determinação dos padrões de produção e deposição de serapilheira, provavelmente em razão da origem dos fragmentos e das várias décadas já decorridas desde sua regeneração. A conectividade das manchas de mata indicou a importância na manutenção das espécies arbóreas zoocóricas e, consequentemente, de fluxos de animais dispersores.

Embora os padrões aqui observados não possam ser generalizados porque o delineamento seguido não contou com réplicas, este estudo mostra que resultados interessantes podem ser obtidos relacionando-se padrões da paisagem a padrões e processos ecológicos. Essa abordagem - que considera a influência da estrutura da paisagem em padrões de produção de serapilheira - é ainda muito pouco explorada no Brasil, tanto que estes autores desconhecem tais trabalhos para florestas extraAmazônicas. Mais estudos com este enfoque devem ser conduzidos, procurando-se replicar os fragmentos para a obtenção de resultados mais conclusivos.

Agradecimentos - Os autores são gratos à FAPESP (Fundação de Amparo à Pesquisa do Estado de São Paulo) pelo auxílio financeiro concedido ao projeto "Conservação da Biodiversidade em Paisagens Fragmentadas no Planalto Atlântico de São Paulo" (processos no 99/05123-4, Projeto Biota), do qual este trabalho foi parte. Também agradecem o auxílio em campo prestado pelos técnicos Paulo César Fernandes e Maurício Perine e pelas alunas Daniela Petenon, Regina Alonso e Flávia Moraes de Jesus, que ajudaram na triagem do material. Dr. Marcio Martins prestou grande auxílio na edição final das figuras.

\section{Referências bibliográficas}

ASHTON, P.M.S. 1992. Some measurements of the microclimate within a Sri Lankan tropical rainforest. Agricultural and Forest Meteorology 59:217-235.

BENITEZ-MALVIDO, J. 1998. Impact of forest fragmentation on seedling abundance in a tropical rain forest. Conservation Biology 12:380-389.

BERNACCI, L.C., FRANCO, G.A.D.C., ARBOCZ, G., CATHARINO, E.L., DURIGAN, G., METZGER, J.P. 2006. $O$ efeito da fragmentação florestal na composição e riqueza de árvores na região da Reserva Morro Grande (Planalto de Ibiúma, SP). Revista do Instituto Florestal 18: $121-166$

BROWN, S. \& LUGO, A.L. 1990. Tropical secondary forests. Journal of Tropical Ecology 6:1-32.

CESAR, O. 1993. Produção de serapilheira na mata mesófila semidecídua da Fazenda Barreiro Rico, Município de Anhembi, SP. Revista Brasileira de Biologia 53:671681.

DELITTI, W.B.C. 1995. Estudos de ciclagem de nutrientes: instrumentos para a análise funcional de ecossistemas terrestres. Oecologia Brasiliensis 1:469-486.

DEVELEY, P.F. \& METZGER, J.P. 2006. Emerging threats to birds in Brazilian Atlantic forests: the roles of forest loss and configuration in a severely fragmented ecosystem. In Emerging threats to tropical forests. (W.F. Laurance \& C.A. Peres, eds.). University of Chicago Press, Chicago, p. 269-290.

DIDHAM, R.K. \& LAWTON, J.H. 1999. Edge structure determines the magnitude of changes in microclimate and vegetation structure in tropical forest fragments. Biotropica 31:17-30. 
DOMINGOS, M., MORAES, R.M., VUONO, Y.S. \& ANSELMO, C.E. 1997. Produção de serapilheira e retorno de nutrientes em um trecho de Mata Atlântica secundária, na Reserva Biológica de Paranapiacaba, SP. Revista Brasileira de Botânica 20:91-96.

EWEL, J.J. 1976. Litter fall and leaf decomposition in a tropical forest succession in eastern Guatemala. Journal of Ecology 64:293-308.

FACELLI, J.M. \& PICKETT, S.T.A. 1991. Plant litter: its dynamics and effects on plant community structure. The Botanical Review 57:1-32.

FAHRIG, L. 2003. Effects of habitat fragmentation on biodiversity. Annual Review of Ecology, Evolution and Systematics 34:487-515.

FOX, B.J., TAYLOR, J.E., FOX, M.D. \& WILLIAMS, C. 1997. Vegetation changes across edges of rainforest remnants. Biological Conservation 82:1-13.

GASCON, C. WILLIAMSON, G.B. \& FONSECA, G.A.B. 2000. Receding forest edges and vanishing reserves. Science 288:1356-1358.

GOLLEY, F.B., MCGINNIS, J.T., CLEMENTS, R.G., CHILD, G.I. \& DUEVER, M.J. 1978. Ciclagem de minerais em um ecossistema de floresta tropical úmida. EPU/EDUSP, São Paulo.

GREEN, P.T. 1998. Litterfall in rain forest on Christmas Island, Indian Ocean: quantity, seasonality, and composition. Biotropica 30:671-676.

HILL, J.L \& CURRAN, P.J. 2003. Area, shape and isolation of tropical forest fragments: effects on tree species diversity and implications for conservation. Journal of Biogeography 30:1391-1403.

INSTITUTO AGRONÔMICO DE CAMPINAS-CIIAGRO (Centro Integrado de Informação Meteorológica) s/data. CIIAGRO on line. Governo do Estado de São Paulo, São Paulo. Disponível em: http://www.iac.sp.gov.br. (Acesso em 26/04/2003.)

JORDAN, C.F. 1985. Nutrient cycling in tropical forest ecosystems. John Wiley \& Sons, Reino Unido.

KAPOS, V. 1989. Effects of isolation on the water status of forest patches in the Brazilian Amazon. Journal of Tropical Ecology 5:173-185.

KÖPPEN, W. 1948. Climatologia: con un estudio de los climas de la tierra. Fundo de Cultura Econômica. México.

LAURANCE, W.F. 1991. Edge effects in tropical forest fragments: application of a model for the design of nature reserves. Biological Conservation 57:205-219.

LAURANCE, W.F., FERREIRA, L.V., MERONA, J.R. \& LAURANCE, S.G. 1998. Rain forest fragmentation and the dynamics of Amazonian tree communities. Ecology 79:2032-2040.

LAURANCE, W.F., LOVEJOY, T.E., VASCONCELOS, H.L., BRUNA, E.M., DIDHAM, R.K., STOUFFER,P.C., GASCON, C., BIERREGAARD, R.O.,LAURANCE, S.G. \& SAMPAIO, E. 2002. Ecosystem decay of Amazonian forest fragments: a 22-year investigation. Conservation Biology 16:605-618.
LOVEJOY, T.E., RANKIN, J.M., BIERREGAARD, R.O., BROWN, K.S., EMMONS, L.H., VANDER VOORT, M.E. 1984. Ecosystem decay of Amazon forest fragments. In Extinctions (M.H. Nitecki, ed.). University of Chicago Press, Chicago, p.296-325.

McGARIGAL, K. \& MARKS, B.J. 1995. FRAGSTATS: Special Pattern Analysis Program for Quantifying Landscape Structure. Department of Agriculture, Forest Service, Pacific Northwest Research Station (Gen. Tech. Rep. PNW-GTR, 351), Portland, U.S.

MORAES, R.M. 1993. Ciclagem de nutrientes minerais em mata atlântica de encosta e mata sobre restinga, na Ilha do Cardoso, Cananéia, SP: produção de serapilheira e transferência de nutrientes. Dissertação de Mestrado, Instituto de Biociências, Universidade de São Paulo, São Paulo.

MORAES, R.M. 2002. Ciclagem de nutrientes na floresta do PEFI: produção e decomposição da serapilheira. In Parque Estadual das Fontes do Ipiranga, unidade de conservação que resiste à urbanização de São Paulo. (D. Bicudo, M. Forti \& C. Bicudo, eds). Secretaria de Estado do Meio Ambiente, São Paulo, p.133-142.

MORAES, R.M., DELITTI, W.B.C. \& VUONO, Y.S. 1999. Litterfall and litter nutrient content in two Brazilian Tropical Forests. Revista Brasileira de Botânica 22:9-16.

MURCIA, C. 1995. Edge effects in fragmented forests: implications for conservation. Trends in Ecology \& Evolution 10:58-62.

OLIVEIRA-FILHO, A.T., MELLO, J.M. \& SCOLFORO, J.R. 1997. Effects of past disturbance and edges on tree community structure and dynamics within a fragment of tropical semideciduous forest in south-eastern Brazil over a five-year period (1987-1992). Plant Ecology 131:45-66.

PARDINI, R., SOUZA, S.M., BRAGA-NETO, R. \& METZGER, J.P. 2005. The role of forest structure, fragment size and corridors in maintaining small mammal abundance and diversity in an Atlantic forest landscape. Biological Conservation 124: 253-266.

PIVELLO, V.R., PETENON, D., JESUS, F.M., MEIRELLES, S.T., VIDAL, M.M., ALONSO, R.A.S., FRANCO, G.A.D.C. \& METZGER, J.P. 2006. Chuva de sementes em fragmentos de floresta Atlântica (São Paulo, SP), sob diferentes situações de conectividade, estrutura florestal e proximidade à borda. Acta Botanica Brasilica 20:845-859.

ROSS, J. L. S. \& MOROZ, I. C. 1997. Mapa Geomorfológico do Estado de São Paulo, escala 1:500.000. GeografiaFFLCH-USP, IPT/FAPESP, São Paulo, vol. 1.

SCHLITTLER, F.H.M., MARINIS, G. \& CÉSAR, O. 1993. Produção de serapilheira na floresta do Morro do Diabo, Pontal do Paranapanema - SP. Naturalia 18:135-147.

SEABRA, M. 1971. Vargem Grande: Organização e Transformações de um Setor do Cinturão Verde Paulistano. Tese de Doutorado, Instituto de Geografia, Universidade de São Paulo, São Paulo. 
SILVA, W.G., METZGER, J.P., SIMÕES, S. \& SIMONETTI, C. 2007. Relief influence on the spatial distribution of the Atlantic Forest cover on the Ibiúna Plateau, SP. Brazilian Journal of Biology 67:631-637.

SIZER, N.C., TANNER, E.V.J. \& FERRAZ, I.D.K. 2000. Edge effects on litterfall mass and nutrient concentrations in forest fragments in central Amazonia. Journal of Tropical Ecology 16:853-863.

SONGWE, N.C., FASEHUN, F.E. \& OKALI, D.U.U. 1988. Litterfall and productivity in a tropical rain forest, Southern Bankundu Forest, Cameroon. Journal of Tropical Ecology 4:25-37.

TABARELLI, M., MANTOVANI, W. \& PERES, C.A. 1999. Effects of habitat fragmentation on plant guild structure in the montane Atlantic Forest of southeastern Brazil. Biological Conservation 91:119-127.
VELOSO, H.P., RANGEL FILHO, A.L.R. \&. LIMA, J.C.A. 1991. Classificação da Vegetação Brasileira Adaptada a um Sistema Universal. IBGE, Rio de Janeiro.

VENEKLAAS, E.J. 1991. Litterfall and nutrient fluxes in two montane tropical rain forests, Colômbia. Journal of Tropical Ecology 7:319-336.

VITOUSEK, P.M. \& SANFORD, R.L.Jr. 1986. Nutrient cycling in moist tropical forest. Annual Review of Ecology and Systematics 17:137-167.

WERNECK, M.S., PEDRALLI, G. \& GIESEKE, L.F. 2001. Produção de serapilheira em três trechos de uma floresta semidecídua com diferentes graus de perturbação na Estação Ecológica do Tripuí, Ouro Preto, MG. Revista Brasileira de Botânica 24:195-198.

ZAR, J.H. 1999. Biostatistical Analysis. Prentice Hall, New Jersey. 